time standing than those with CP (CP Median 2.99 hours, $n=7$; ASD Median 4.3 hours, $n=6 ; U=7.000, z=-2.00$, $P=0.045)$. TD children took more steps than those with CP or DCD (TD Median 9873, $n=8$; CP Median 6714, $n=7$; $U=2.00, z=-3.01 ; P=0.003$ ), (TD Median 9873, $n=8$; DCD Median 5557, $n=7 ; U=2.00, z=-2.38 ; P=0.017)$.

Discussion and conclusions: Results provide preliminary evidence that PA levels vary across disability populations. Children with CP have similar PA levels to those of TD children; however children with ASD and DCD appear to be less active.

Impact and implications: There is a need for interventions to increase PA levels across all disability populations, such interventions need to be population specific.

Funding acknowledgement: No funding.

http://dx.doi.org/10.1016/j.physio.2016.10.326

POS271

\section{Burns prevention in children under 5 years: increasing health literacy}

G. Colaço*, J. Cadaixa

\section{Escola Superior de Saúde - Instituto \\ Politécnico de Setúbal, Physiotherapy, Setúbal, Portugal}

Relevance: Burns leave severe impairments, sometimes permanent, for those involved. In Portugal, burns are the second cause of accidental death in children aged 5 years old and younger. These could be prevented in $90 \%$ of the cases but unfortunately the number of burns in children within this age range has been recently increasing. Most of these take place in domestic environments, mainly as a consequence of contact with boiling liquids or thermic sources (such as electric or gas heaters). The low levels of knowledge regarding risky situations and respective prevention measures are two of the factors which contribute to the present prevalence of burns in young children.

Purpose: To develop in children themselves, the knowledge to engage in preventive behaviours which reduce the risk of burns in the domestic settings and simultaneously to promote the adequate use of the technique "Stop, Lie \& Role" in a situation of fire burn.

Approach/evaluation: This project involved the development of a programme which consisted of three sessions implemented directly with children. Each of these sessions included two components, a more theoretical and a more practical one. Three schools of the region of Setúbal were approached and two agreed to collaborate. In total 25 children volunteered (with own and parents' consent) to participate in the Project. A questionnaire was developed to assess the knowledge about possible burning agents and knowledge about preventive behaviours. A check list was used to evaluate the adequacy of the educational materials and a second one was also developed to verify the adequate use of the "Stop, Lie \& Role".

Outcomes: The programme implemented was inspired by those of Moore et al. (2004) and by Wood et al. (2010). Motivational strategies of Atiyeh et al. (2009), and posititve reinforcement of Gielen, Borzekowski \& Rimal (2010) were included. At the end of the programme all children identified correctly 13 of the 16 most common agents that cause burns at home. In addition, $86 \%$ of the children identified correctly the 4 agents which may cause burns and $94 \%$ identified correctly the preventive behaviours to adopt in four distinct burns.

Discussion and conclusions: All pre-school children recognized at least 13 possible causes of burning, out of the 16 explored. The activities implemented in each session have revealed themselves as effective in the acquisition of knowledge by the children, with clear messages and goals being attained. The educational materials seem to be adequate to this particular population. Other programmes suggest that these interventions may be a significant step for the education and increased health literacy of children, enabling them to become active agents in the prevention of burns.

Impact and implications: The results obtained seem promising for continuation of the project in the other schools of the district. Projects such as the present one may be an effective and sustainable way of implementing safer behaviours in children, contributing to the diminishing of burn related costs, for families and society.

Funding acknowledgement: This project had no funding.

http://dx.doi.org/10.1016/j.physio.2016.10.327

POS272

Learning and influencing in a digital age is physiotherapy making (mega) byte-sized progress?

\section{Ellis}

Leeds Beckett University, Faculty of Health and Social Sciences, Leeds, United Kingdom

Relevance: This presentation has relevance to the following three themes:

- Policy, strategy and influencing

- Research education and practice

- Practice in a digital age

Purpose: In this presentation I will explore and challenge physiotherapists to reflect on the extent to which they and their profession are keeping pace in using and embracing digital technologies as tools for supporting learning and development (pre-registration and for continuing professional development) and as tools for strategic influencing and raising the profile and impact of the profession.

As a UK physiotherapist with a 28 year leadership career in higher education and as a Professor in Assessment, Learning 